\title{
Modelos de Onsager e Debye para fluidos polares puros
}

\author{
Onsager and Debye models for pure polar fuids
}

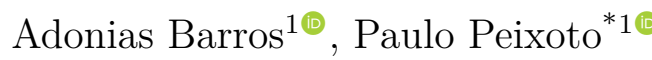 \\ ${ }^{1}$ Universidade Federal de Pernambuco, Núcleo de Formação Docente, Caruaru, PE, Brasil.
}

Recebido em 21 de abril de 2020. Revisado em 01 de junho de 2020. Aceito em 10 de junho de 2020.

Em 1936, Lars Onsager obteve uma equação que relaciona a constante dielétrica de fluidos polares puros ao momento de dipolo permanente de suas moléculas constituintes, e também ao índice de refração, à densidade e à temperatura do meio. Neste artigo, guiamos o leitor ou a leitora à obtenção da equação de Onsager, demandando dele ou dela, contudo, conhecimentos elementares de eletrostática em meios dielétricos e de mecânica estatística clássica - particularmente do ensemble canônico -, ambos ao nível de um curso de graduação em física. No percurso - e isso é o que mais nos interessa -, discutimos modelagem em física. Em uma seção adicional, discutimos o modelo de Debye como resultado de uma aproximação de campo médio aplicada ao modelo de Onsager, e apresentamos o modelo de Clausius-Mossotti como caso particular dos modelos de Debye e Onsager. Sugestão de uso deste texto: no estudo ou ensino de tópicos complementares em disciplinas de eletrodinâmica clássica, mecânica estatística ou físico-química, em cursos de graduação ou pós-graduação.

Palavras-chave: modelo de Onsager, modelo de Debye, modelo de Clausius-Mossotti, constante dielétrica, dielétricos.

In 1936, Lars Onsager obtained an equation that relates the dielectric constant of pure polar fluids to the permanent dipole moment of its constituent molecules, and also to the refractive index, the density and the temperature of the medium. In this paper, we guide the reader to find the Onsager equation, demanding from him or her, however, basic knowledge on electrostatic in matter and on classical statistical mechanics - particularly on the canonical ensemble -, both at an undergraduate level. Along the way - and this is our main interest in this work - we discuss modeling in physics. In an additional section, we discuss the Debye model as resulting from a mean field approximation applied to the Onsager model, and we show the Clausius-Mossotti model as a particular case of the Debye and Onsager models. Suggested use of this text: in the study or teaching of additional topics in undergraduate or graduate courses in classical electrodynamics, statistical mechanics, or physical chemistry.

Keywords: Onsager model, Debye model, Clausius-Mossotti model, dielectric constant, dielectrics.

\section{Preliminares}

A equação de Onsager 1$], 1]$

$$
\frac{\left(\epsilon_{r}-n^{2}\right)\left(2 \epsilon_{r}+n^{2}\right)}{\epsilon_{r}\left(n^{2}+2\right)^{2}}=\frac{N}{3 \epsilon_{0}} \frac{p_{0}^{2}}{3 k T},
$$

relaciona a constante dielétrica $\epsilon_{r}$ de um fluido polar puro ao módulo $p_{0}$ do momento de dipolo permanente de suas moléculas constituintes, e também ao índice de refração $n$, à densidade numérica $N$ (número de moléculas por unidade de volume) e à temperatura absoluta $T$ do meio ${ }^{2}$ $k$ é a constante de Boltzmann, e $\epsilon_{0}$ é a permissividade elétrica do vácuo. Trata-se de uma equação bastante conhecida na área de teoria de polarização elétrica, e neste artigo guiaremos o leitor ou a leitora à sua obtenção. Discutiremos também o modelo de Debye como resultado

*Endereço de correspondência: phrpeixoto@yahoo.com.br

${ }^{1}$ Neste artigo, escrevemos a equação de Onsager no Sistema Internacional de Unidades (SI). Ela foi originalmente expressa no sistema gaussiano 1].

${ }^{2}$ Como veremos, o índice de refração pode ser relacionado à polarizabilidade $\alpha$ das moléculas do fluido. de uma aproximação de campo médio aplicada ao modelo de Onsager, e apresentaremos o modelo de ClausiusMossotti como caso particular dos modelos de Debye e Onsager.

São pré-requisitos, para uma boa compreensão deste artigo, conhecimentos de eletrostática em meios dielétricos ao nível do capítulo 4 do Griffiths [2], e de mecânica estatística clássica - particularmente do ensemble canônico - ao nível do capítulo 7 do Reif [3]. Adicionalmente, um conhecimento elementar sobre ondas eletromagnéticas no vácuo e em meios materiais lineares - ao nível das seções 9.2.1 e 9.3.1 do Griffiths, respectivamente - é bem vindo, embora não seja indispensável. Contudo, mesmo assumindo atendidos esses pré-requisitos, revisaremos nesta seção alguns tópicos fundamentais para uma boa compreensão do modelo de Onsager.

Considere uma distribuição localizada de cargas em torno da origem de um sistema de coordenadas. Conhecida essa distribuição, o potencial elétrico $V(\boldsymbol{r})$ gerado pela mesma em um ponto $\boldsymbol{r}$ qualquer pode ser expresso 
formalmente como um somatório ou uma integral. Em algumas situações, pode ser interessante obtermos uma expressão aproximada para esse potencial - especialmente se o módulo $r$ do vetor $\boldsymbol{r}$ é bem maior que os comprimentos que caracterizam a distribuição de cargas. Se a carga líquida $Q$ do sistema não é nula, em primeira aproximação temos o potencial de uma carga puntiforme $Q$ localizada na origem: $Q / 4 \pi \epsilon_{0} r$. Mas sendo $Q=0$, a primeira aproximação para $V(\boldsymbol{r})$ é

$$
V(\boldsymbol{r}) \approx V_{\mathrm{dip}}(\boldsymbol{r}) \equiv \frac{1}{4 \pi \epsilon_{0}} \frac{\boldsymbol{p} \cdot \hat{r}}{r^{2}}
$$

contanto que $\boldsymbol{p} \equiv \sum_{i} q_{i} \boldsymbol{r}_{i}^{\prime}$ - o chamado momento de dipolo da distribuição de cargas - não seja nulo. Aqui, $\boldsymbol{r}_{i}^{\prime}$ é a posição da carga $q_{i} !^{3}$

O exemplo mais simples de uma distribuição de cargas com $Q=0$ e $\boldsymbol{p} \neq 0$ é o que chamamos de dipolo elétrico físico (ou, simplesmente, dipolo elétrico ou dipolo físico): duas cargas puntiformes $q>0$ e $-q$ separadas por uma distância $d$. Neste caso, obtemos $\boldsymbol{p}=q \boldsymbol{d}$, em que $\boldsymbol{d}$ é o vetor que vai da carga negativa à carga positiva, sendo $d$ seu módulo. É interessante observar que o conceito de dipolo físico pode ser usado mesmo para distribuições bem mais complexas, contanto que a carga líquida seja nula e que o centro de carga positiva, $\boldsymbol{r}_{+} \equiv \sum_{i} q_{i(+)} \boldsymbol{r}_{i(+)}^{\prime} / \sum_{i} q_{i(+)}$, não coincida com o centro de carga negativa, $\boldsymbol{r}_{-} \equiv \sum_{i} q_{i(-)} \boldsymbol{r}_{i(-)}^{\prime} / \sum_{i} q_{i(-)}{ }^{4}$ Continuamos obtendo $\boldsymbol{p}=q \boldsymbol{d}$, mas com as expressôes mais gerais $q=\sum_{i} q_{i(+)},-q=\sum_{i} q_{i(-)}$ e $\boldsymbol{d}=\boldsymbol{r}_{+}-\boldsymbol{r}_{-}$; ou seja, $q$ é a carga positiva total, $-q$ é a carga negativa total e $\boldsymbol{d}$ é o vetor que vai do centro de carga negativa ao centro de carga positiva. Mas mesmo para um dipolo físico propriamente dito (ou seja, constituído de apenas duas cargas puntiformes), a expressão em (1) é uma aproximação - embora uma ótima aproximação se $r \gg d$ (considerando o dipolo centrado na origem do sistema de coordenadas). Contudo, podemos tornar a aproximação em (1) exata introduzindo o conceito de dipolo puro.

$\mathrm{O}$ conceito de dipolo puro (ou dipolo elétrico puro) é tão legítimo quanto o conceito de carga puntiforme. Podemos definir um dipolo puro de momento de dipolo $\boldsymbol{p}$ como o limite de um dipolo físico quando $d$ tende a 0 , $q$ tende a $\infty$, mas o produto $q d$ tende a $p \equiv|\boldsymbol{p}|$. Trata-se, é claro, de uma idealização, mas que é bastante útil para o desenvolvimento da eletrodinâmica de meios contínuos.

Podemos agora imaginar uma distribuição contínua de dipolos puros (assim como podemos imaginar, em uma análise macroscópica, uma distribuição contínua de carga elétrica, mesmo sabendo que a carga elétrica é quantizada) e definir o vetor polarização elétrica $\boldsymbol{P}\left(\boldsymbol{r}^{\prime}\right)$ como o momento de dipolo elétrico por unidade de volume


ser substituído por uma integral.

${ }^{4}$ Estamos fazendo uso, aqui, da seguinte notação: $q_{i(+)}=q_{i}$, se $q_{i}>0$; caso contrário, $q_{i(+)}=0$ (e por isso podemos escrever $\boldsymbol{r}_{i(+)}^{\prime}=\mathbf{r}_{i}^{\prime}$. Analogamente, $q_{i(-)}=q_{i}$, se $q_{i}<0$; caso contrário, $q_{i(-)}=0$ (e por isso podemos escrever $\boldsymbol{r}_{i(-)}^{\prime}=\mathbf{r}_{i}$ ). Esta nota é para os puristas; acreditamos que a notação é autoexplicativa.
}

no ponto $\boldsymbol{r}^{\prime} 5^{5}$ Então o momento de dipolo elétrico em um volume infinitesimal $\mathrm{d} \tau^{\prime}$ centrado no ponto $\boldsymbol{r}^{\prime}$ é $\mathrm{d} \boldsymbol{p}=$ $\boldsymbol{P} \mathrm{d} \tau^{\prime}$, e o potencial elétrico gerado por esse momento de dipolo infinitesimal no ponto $\boldsymbol{r}$ é $\mathrm{d} V=\boldsymbol{P} \mathrm{d} \tau^{\prime} \cdot \hat{\boldsymbol{\kappa}} / 4 \pi \epsilon_{0} \boldsymbol{r}^{2}$, em que $\boldsymbol{r} \equiv \boldsymbol{r}-\boldsymbol{r}^{\prime}$ (veja a Fig. 1). Integrando sobre a distribuição de dipolos puros (domínio $\mathscr{D}$ ), obtemos o potencial elétrico $V(\boldsymbol{r})$ gerado pela mesma em um ponto $\boldsymbol{r}$ externo (veja a Fig.1):

$$
V(\boldsymbol{r})=\frac{1}{4 \pi \epsilon_{0}} \int_{\mathscr{D}} \frac{\boldsymbol{P} \cdot \hat{\boldsymbol{\gamma}}}{\boldsymbol{r}^{2}} \mathrm{~d} \tau^{\prime} .
$$

Trabalhando com o conceito de dipolo puro e com a eletrodinâmica de meios contínuos, esta expressão é exata; não se trata de uma aproximação. E - o que é mais interessante - ela continua exata mesmo considerando o ponto $\boldsymbol{r}$ interno à distribuição.

A afirmativa de que a expressão em (2) continua exata mesmo considerando o ponto $\boldsymbol{r}$ interno à distribuição D de dipolos puros (veja a Fig. 1) não deve causar estranheza. Você certamente já calculou o campo elétrico em um ponto $\boldsymbol{r}$ no interior de uma esfera de raio $R$ uniformemente carregada, centrada na origem do sistema de coordenadas, concluindo que ele tem direção radial e que seu módulo varia linearmente com a distância $r$ do ponto considerado à origem (para $r<R$, é claro). $\mathrm{O}$ que precisa ficar claro é que, seja para uma distribuição contínua de carga elétrica ou de dipolos puros, modelando um sistema real, o campo em um ponto $\boldsymbol{r}$ interno à distribuição é um campo macroscópico. Não faz sentido pensarmos que se trata do campo que atua sobre uma determinada molécula do material, ou em parte dela. Se tivéssemos trabalhando no nível de átomos ou moléculas, não poderíamos, em primeiro lugar, considerar uma distribuição contínua, seja de carga elétrica ou de dipolos puros. Além do mais, sabemos que no nível atômico ou molecular a eletrodinâmica clássica não é suficiente para descrever a matéria. O que devemos ter em mente é que se uma pequena carga de prova $q$ for posta em um ponto da distribuição em que o campo elétrico macroscópico é

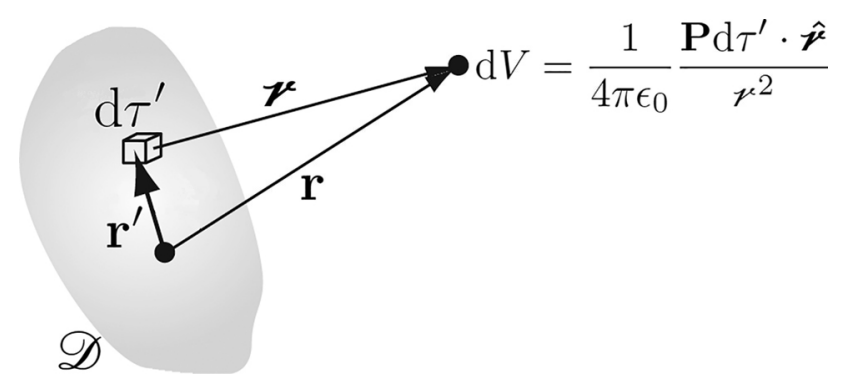

Figura 1: Distribuição contínua $\mathscr{D}$ de dipolos puros. O potencial gerado por essa distribuição no ponto $r$ é expresso pela igualdade (2), com $\boldsymbol{P}=\boldsymbol{P}\left(\boldsymbol{r}^{\prime}\right)$.

\footnotetext{
${ }^{5}$ Trata-se da grandeza correspondente à densidade volumétrica de carga, $\rho\left(\boldsymbol{r}^{\prime}\right)$, no caso de uma distribuição contínua de dipolos puros, em vez de carga elétrica (ou seja, monopolos). Mas, é claro, enquanto $\rho$ é uma grandeza escalar, $\boldsymbol{P}$ é uma grandeza vetorial.
} 
$\boldsymbol{E}$, a força elétrica sobre ela será $\boldsymbol{F}=q \boldsymbol{E}{ }^{6}$ Se de alguma forma quisermos considerar a estrutura atômica da matéria, teremos que pensar esse campo macroscópico como um campo médio sobre uma porção de muitos milhares de moléculas (que, do ponto de vista macroscópico, ocupa um volume que pode ser considerado infinitesimal, e que poderia ser ocupado por nossa carga de prova). E é justamente aí que reside um dos aspectos mais importantes do desenvolvimento do modelo de Onsager: como veremos na próxima seção, precisaremos obter uma expressão para o campo que atua em uma determinada molécula do dielétrico (gás ou líquido) - o chamado campo interno -, mas a eletrodinâmica de meios contínuos não nos dá essa expressão; é preciso modelar!

Voltando à igualdade (2), através de uma manipulação matemática relativamente simples $[2]$ podemos reescrevêla como

$$
V=\frac{1}{4 \pi \epsilon_{0}}\left[\int_{\mathscr{D}} \nabla^{\prime} \cdot\left(\frac{\boldsymbol{P}}{\mu}\right) \mathrm{d} \tau^{\prime}-\int_{\mathscr{D}} \frac{1}{\mu}\left(\nabla^{\prime} \cdot \boldsymbol{P}\right) \mathrm{d} \tau^{\prime}\right],
$$

em que $\nabla^{\prime}$. significa que a divergência é calculada com relação às coordenadas que constituem $\boldsymbol{r}^{\prime}$. Fazendo uso do teorema da divergência na primeira integral, obtemos

$$
V=\frac{1}{4 \pi \epsilon_{0}}\left[\oint_{S} \frac{1}{\mu} \boldsymbol{P} \cdot \hat{\boldsymbol{n}}^{\prime} \mathrm{d} a^{\prime}+\int_{\mathscr{D}} \frac{1}{\mu}\left(-\nabla^{\prime} \cdot \boldsymbol{P}\right) \mathrm{d} \tau^{\prime}\right],
$$

em que $S$ é a superfície que constitui a fronteira da distribuição $\mathscr{D}$ de dipolos, $\hat{\boldsymbol{n}}^{\prime}$ é um versor normal a $S$ no ponto $\boldsymbol{r}^{\prime}$ (apontando para fora da região $\mathscr{D}$ ) e d $a^{\prime}$ é um elemento de área de $S$ em torno de $\hat{\boldsymbol{n}}^{\prime}$. A beleza desse desenvolvimento é que temos no primeiro e no segundo termos do segundo membro da igualdade (4) (após as multiplicações das integrais por $1 / 4 \pi \epsilon_{0}$ ) as formas matemáticas, respectivamente, de um potencial gerado por uma superfície carregada com densidade superficial de carga $\sigma_{b}\left(\boldsymbol{r}^{\prime}\right)=\boldsymbol{P}\left(\boldsymbol{r}^{\prime}\right) \cdot \hat{\boldsymbol{n}}^{\prime}$ e de um potencial gerado por uma distribuição volumétrica de carga com densidade $\rho_{b}\left(\boldsymbol{r}^{\prime}\right)=-\nabla^{\prime} \cdot \boldsymbol{P}\left(\boldsymbol{r}^{\prime}\right)$. Ou seja, o potencial $V(\boldsymbol{r})$ expresso na igualdade $(2)$, gerado por uma distribuição contínua $\mathscr{D}$ de dipolos puros, é igual ao potencial produzido por uma distribuição superficial de carga, na fronteira $S$ da região $\mathscr{D}$, com densidade superficial (agora omitindo o ". ")

$$
\sigma_{b}=\boldsymbol{P} \cdot \hat{\boldsymbol{n}},
$$

e por uma distribuição volumétrica de carga, na região $\mathscr{D}$, com densidade volumétrica

$$
\rho_{b}=-\nabla \cdot P
$$

Essas cargas - chamadas de cargas ligadas - têm interpretação física como acúmulos de carga em uma distribuição de dipolos (como explica Griffiths na seção 4.2.2 de seu livro [2], considerando pequenos dipolos físicos).

Podemos então separar, na lei de Gauss (em sua forma diferencial), a densidade $\rho$ de cargas elétricas em dois

\footnotetext{
${ }^{6}$ Estamos supondo, aqui, que a presença da carga de prova não altera a distribuição; ou então devemos fazer $q \rightarrow 0$.
}

termos: a densidade $\rho_{f}$ de cargas livres e a densidade $\rho_{b}$ de cargas ligadas ${ }^{7}$ Compreenda que a expressão "cargas livres" não está sendo usada aqui na acepção encontrada no estudo de condutores elétricos. Aqui, "carga livre" é qualquer carga elétrica que não possa ser interpretada como acúmulo de carga em uma distribuição de dipolos. Por exemplo, imagine pequeninas esferas com carga elétrica não-nula mergulhadas em um líquido dielétrico. Dizemos que a carga elétrica em cada uma dessas esferinhas é uma carga livre. Mas observe que essas esferas podem ter suas posições de alguma forma fixadas, e, portanto, o termo "cargas livres", usado aqui, deve ser bem compreendido.

Pois bem, fazendo uso da igualdade (6) na lei de Gauss, obtemos:

$$
\begin{gathered}
\boldsymbol{\nabla} \cdot \boldsymbol{E}=\frac{\rho}{\epsilon_{0}} \Rightarrow \\
\epsilon_{0} \boldsymbol{\nabla} \cdot \boldsymbol{E}=\rho_{f}+\rho_{b}=\rho_{f}-\nabla \cdot \boldsymbol{P} \Rightarrow \\
\boldsymbol{\nabla} \cdot\left(\epsilon_{0} \boldsymbol{E}+\boldsymbol{P}\right)=\rho_{f} .
\end{gathered}
$$

Se $\boldsymbol{P}$ é proporcional a $\boldsymbol{E}$, caracterizando o meio como linear, podemos escrever:

$$
\boldsymbol{P}=\epsilon_{0} \chi_{e} \boldsymbol{E}
$$

em que $\chi_{e}$ é a chamada susceptibilidade elétrica do meio 8 Substituindo a igualdade (9) na igualdade (8) obtemos, para um meio linear:

$$
\begin{gathered}
\epsilon_{0}\left(1+\chi_{e}\right) \boldsymbol{\nabla} \cdot \boldsymbol{E}=\rho_{f} \Rightarrow \\
\boldsymbol{\nabla} \cdot \boldsymbol{E}=\frac{\rho_{f}}{\epsilon}
\end{gathered}
$$

sendo $\epsilon \equiv \epsilon_{0}\left(1+\chi_{e}\right)$ a denominada permissividade elétrica do meio (lembre-se que $\epsilon_{0}$ é a permissividade elétrica do vácuo). A permissividade relativa $\epsilon_{r}$, ou constante dielétrica, do material, é assim definida:

$$
\epsilon_{r} \equiv \frac{\epsilon}{\epsilon_{0}}=1+\chi_{e}=1+\frac{P}{\epsilon_{0} E},
$$

e, como a susceptibilidade elétrica, é uma medida de quão polarizável é um dielétrico.

Deve ficar claro que o campo $\boldsymbol{E}$, em $(10)$, é um campo macroscópico ${ }^{9}$ E a igualdade $(10)$, que é matematicamente idêntica à lei de Gauss em sua forma geral (igualdade (7), pode ser usada para o cálculo do campo macroscópico $\boldsymbol{E}$ gerado por uma distribuição (discreta ou contínua) de carga livre em um meio dielétrico linear de permissividade elétrica $\epsilon$, desprezados efeitos de borda (do dielétrico). Todo o efeito da polarização do meio fica incorporado no valor de $\epsilon$ (ou, equivalentemente, de $\epsilon_{r}$ ) o que é impressionante, não acha?

\footnotetext{
${ }^{7}$ Perceba que estamos usando aqui as iniciais das palavras "free" e "bound". Essa foi nossa escolha porque as palavras "livres" e "ligadas" começam com a mesma letra.

${ }^{8} \mathrm{~A}$ linearidade de um dielétrico depende não apenas do material, mas da intensidade do campo elétrico.

${ }^{9}$ Se você quiser aprofundar a discussão sobre campos macroscópicos em um meio dielétrico, veja, por exemplo, a excelente discussão feita pelo Griffiths na seção 4.2.3 (The Field Inside a Dielectric) de seu livro 2].
} 


\section{Roteiro para obtenção da equação de Onsager}

O ponto de partida para a obtenção da equação de Onsager é a relação

$$
\epsilon_{r}=1+\frac{P}{\epsilon_{0} E}
$$

obtida na seção anterior para um meio dielétrico linear.

Considere que o nosso meio dielétrico linear é um fluido polar puro - ou seja, um gás ou um líquido constituído de moléculas polares de um único tipo. $\mathrm{O}$ momento de dipolo $\boldsymbol{p}$ de uma dada molécula do fluido, em um certo instante, pode ser expresso (até a primeira ordem) como

$$
\boldsymbol{p}=\boldsymbol{p}_{0}+\alpha \boldsymbol{E}_{\mathrm{int}}
$$

em que $\boldsymbol{p}_{0}$ é o momento de dipolo permanente da molécula (que pode variar em direção, mas cujo módulo, $p_{0}$, é constante), $\alpha$ é a sua polarizabilidade e $\boldsymbol{E}_{\text {int }}$ é o chamando campo interno, que é o campo elétrico que age sobre aquela molécula, naquele instante. Para moléculas apolares, temos $p_{0}=0$. O termo $\alpha \boldsymbol{E}_{\text {int }}$ é propriamente chamado de momento de dipolo induzido, e $\boldsymbol{p}$ é então denominado momento de dipolo total. Esse vetor varia constantemente, devido às rotações da molécula e às constantes variações do campo $\boldsymbol{E}_{\text {int }}$. É preciso ficar claro que ao usarmos a igualdade $\boldsymbol{p}=\boldsymbol{p}_{0}+\alpha \boldsymbol{E}_{\text {int }}$ estamos supondo que a expansão de $\boldsymbol{p}\left(\boldsymbol{E}_{\text {int }}\right)$ até a primeira ordem em $\boldsymbol{E}_{\text {int }}$ é suficiente, na modelagem (ou seja, que termos de mais alta ordem são desnecessários, para os campos e o meio considerados), e que o campo $\boldsymbol{E}_{\text {int }}$ praticamente não varia ao longo da molécula - o que não é razoável, por exemplo, para moléculas longas interagindo com moléculas vizinhas próximas.

Muito bem; agora, considere que o nosso fluido polar puro está sanduichado por um capacitor de placas paralelas carregado, e que, portanto, está submetido a um campo elétrico externo $\boldsymbol{E}_{\text {ext }}$ uniforme. Isso resulta em um campo macroscópico $\boldsymbol{E}$ uniforme no fluido (suposto homogêneo), e portanto em uma polarização elétrica $\boldsymbol{P}$ também uniforme (veja a Fig. 2). Vamos considerar um pequeno volume macroscópico $\mathscr{V}$ do fluido (por exemplo, de um milímetro cúbico, ou menos), em uma região qualquer do mesmo. Como o campo vetorial macroscópico $\boldsymbol{P}$ é uniforme, a soma $\boldsymbol{p}_{\mathscr{V}}$ dos momentos de dipolo totais das moléculas que ocupam o volume $\mathscr{V}$ independe de que região do fluido foi escolhida, e é essencialmente constante no tempo, apesar das grandes variações sofridas pelo momento de dipolo de cada molécula, individualmente. Essa constância no tempo se dá porque mesmo o volume $\mathscr{V}$ sendo, do ponto de vista macroscópico, pequeno, é ocupado por um número tão elevado de moléculas que as flutuações estatísticas no vetor $\boldsymbol{p}_{\mathscr{V}}$ são desprezíveis. Em outras palavras, estamos no chamado limite termodinâmico. Podemos então escrever: $\boldsymbol{p}_{\mathscr{V}}=\sum_{i=1}^{\mathcal{N}} \boldsymbol{p}_{i}$, em que $\boldsymbol{p}_{i}$ é o momento de dipolo total da i-ésima molécula (em um determinado instante) e $\mathcal{N}$ é o número total de moléculas no volume $\mathscr{V}$. É interessante reescrevermos:

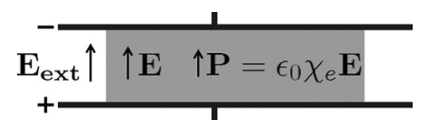

Figura 2: Fluido polar puro sanduichado por um capacitor de placas paralelas carregado. O capacitor aplica no fluido um campo $\boldsymbol{E}_{\text {ext }}$ uniforme, o que resulta em um campo macroscópico $\boldsymbol{E}=\boldsymbol{E}_{\text {ext }} / \epsilon_{r}$ e uma polarização $\boldsymbol{P}$ uniformes no meio. É claro, paredes laterais (não mostradas na figura) mantêm o fluido confinado. E a figura está fora de escala (as placas paralelas estão muito mais próximas; do contrário, o campo $\boldsymbol{E}_{\text {ext }}$ não poderia ser considerado uniforme).

$\boldsymbol{p}_{\mathscr{V}}=\mathcal{N}\left(\sum_{i=1}^{\mathcal{N}} \boldsymbol{p}_{i}\right) / \mathscr{N}=\mathcal{N} \overline{\boldsymbol{p}}$, em que $\overline{\boldsymbol{p}} \equiv\left(\sum_{i=1}^{\mathcal{N}} \boldsymbol{p}_{i}\right) / \mathcal{N}$ é o momento de dipolo molecular médio no volume $\mathscr{V}$. Temos, então: $\boldsymbol{P}=\boldsymbol{p}_{\mathscr{V}} / \mathscr{V}=(\mathscr{N} / \mathscr{V}) \overline{\boldsymbol{p}}$ - ou seja,

$$
\boldsymbol{P}=N \overline{\boldsymbol{p}}
$$

em que $N \equiv \mathcal{N} / \mathscr{V}$ é o número de moléculas por unidade de volume do fluido.

Para calcularmos $\overline{\boldsymbol{p}}$, podemos centrar nossa atenção não no volume $\mathscr{V}$ como um todo, mas em uma única molécula do mesmo, e considerar o restante do fluido como um reservatório térmico. Como você sabe, assim procedendo estaremos trabalhando com o chamado ensemble canônico da mecânica estatística. Podemos imaginar um conjunto enorme de sistemas identicamente preparados (cada sistema sendo constituído da molécula considerada e do restante do fluido), indistinguíveis macroscopicamente, mas distinguíveis em seus microestados. Percorrendo os sistemas do ensemble, a molécula considerada será encontrada em diferentes estados acessíveis, e, escolhendo um sistema ao acaso, a probabilidade $\mathscr{P}_{r}$ de que ela esteja em um determinado estado $r$, de energia $\varepsilon_{r}$, é $\mathscr{P}_{r}=e^{-\beta \varepsilon_{r}} /\left(\sum_{r^{\prime}} e^{-\beta \varepsilon_{r^{\prime}}}\right)$, em que a soma no denominador é feita sobre todos os estados acessíveis à molécula e $\beta=1 / k T$ (sendo $k$ a constante de Boltzmann e $T$ a temperatura do fluido). Segue que $\overline{\boldsymbol{p}}=\sum_{r} \boldsymbol{p}_{r} \mathscr{P}_{r}=\left(\sum_{r} \boldsymbol{p}_{r} e^{-\beta \varepsilon_{r}}\right) /\left(\sum_{r} e^{-\beta \varepsilon_{r}}\right)$, em que $\boldsymbol{p}_{r}$ é o momento de dipolo total da molécula quando ela está no estado $r$, de energia $\varepsilon_{r}$. No cálculo de $\overline{\boldsymbol{p}}$ no modelo de Onsager, esses somatórios são substituídos por integrais, porque, como veremos, as variáveis que determinam o estado $r$ são contínuas. Deve ser bem compreendido que o procedimento acima descrito é válido na medida em que possamos aplicá-lo a qualquer molécula do volume $\mathscr{V}$ inicialmente considerado para o cálculo de $\overline{\boldsymbol{p}}$. A distribuição, nos sistemas do ensemble, dos estados acessíveis à molécula considerada corresponde à distribuição de estados encontrada, num determinado instante, para as moléculas que constituem o volume $\mathscr{V}$.

Para o cálculo do campo interno $\left(\boldsymbol{E}_{\text {int }}\right)$ do modelo de Onsager, a molécula considerada é imaginada em uma cavidade esférica microscópica, e além dessa cavidade o fluido dielétrico é modelado como um meio contínuo (veja a Fig.3). Rigorosamente, isso não faz sentido - especialmente se considerarmos as moléculas mais próximas da molécula na cavidade imaginária. Ou seja, não existe essa cavidade esférica microscópica. No entanto, temos 


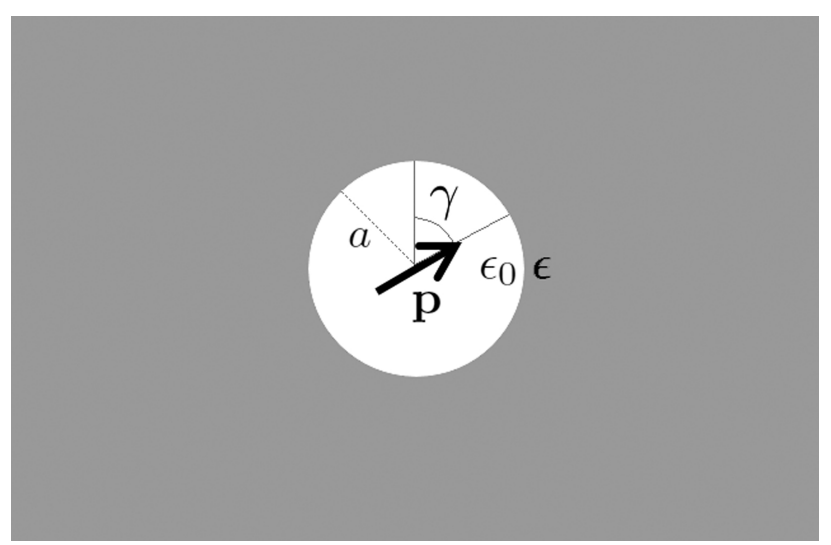

Figura 3: Cavidade esférica microscópica imaginária, de raio $a$, na região central do dielétrico da Fig.2 A cavidade contém uma única molécula do fluido, aqui representada por seu momento de dipolo total $\boldsymbol{p}$ (no instante em que ele forma um ângulo $\gamma$ com o campo externo $\boldsymbol{E}_{\text {ext }}$ aplicado). Além da cavidade, o dielétrico é modelado como um meio contínuo de permissividade $\epsilon$.

aqui um bom exemplo de como os físicos, quando desenvolvem modelos, fazem uso de aproximações que podem até ser consideradas estranhas, mas o que importa é que haja um mínimo de coerência interna e que as previsões do modelo em alguma medida se harmonizem com resultados experimentais. Além do mais, o desenvolvimento de um determinado modelo pode abrir caminho para o desenvolvimento de modelos mais sofisticados. De fato, o modelo de Kirkwood-Fröhlich, que considera interações de curto alcance em fluidos polares puros, foi desenvolvido (inicialmente por Kirkwood [4], tendo recebido depois a contribuição de Fröhlich [5]) a partir do modelo de Onsager.

Obtemos a expressão para o campo interno do modelo de Onsager resolvendo um problema de eletrostática de meios contínuos: calculamos o potencial $V_{\text {cav }}$ no interior da cavidade da Fig. 3 (tratando a cavidade como macroscópica, já que a cavidade microscópica é apenas uma idealização), e então descontamos o potencial gerado pelo próprio dipolo, obtendo $V_{\text {int }}=V_{\text {cav }}-V_{\text {dip }}$, e, em seguida, $\boldsymbol{E}_{\text {int }}=-\nabla V_{\text {int }}$.

Vamos concluir esta seção organizando um roteiro (que usaremos na seção seguinte) para a obtenção da equação de Onsager:

- Passo 1: Calcule o campo interno, $\boldsymbol{E}_{\text {int }}$, resolvendo o problema de eletrostática de meios contínuos apresentado acima.

- Passo 2: Substitua a expressão encontrada para $\boldsymbol{E}_{\text {int }}$ na equação

$$
\boldsymbol{p}=\boldsymbol{p}_{0}+\alpha \boldsymbol{E}_{\mathrm{int}} .
$$

Se $\boldsymbol{E}_{\text {int }}$ for função de $\boldsymbol{p}$, resolva a equação resultante para $\boldsymbol{p}$.

- Passo 3: Usando mecânica estatística, calcule $\overline{\boldsymbol{p}}$, e então obtenha

$$
\boldsymbol{P}=N \overline{\boldsymbol{p}}
$$

- Passo 4: Por fim, substitua o módulo de $\boldsymbol{P}$ (que será proporcional a $E$ ) na igualdade

$$
\epsilon_{r}=1+\frac{P}{\epsilon_{0} E}
$$

Obviamente, esses passos não podem ser bem compreendidos sem o que foi apresentado nesta seção. E no desenvolvimento que leva à equação de Onsager (seção 3 há uma série de detalhes que não estão apresentados nesses passos, nem nesta seção. Mas, essencialmente, a sequência é essa.

\section{Obtenção da equação de Onsager}

Para obtenção da equação de Onsager, seguiremos o roteiro apresentado na seção anterior.

\section{Passo 1}

Obtemos a expressão para o campo interno do modelo de Onsager resolvendo o problema de eletrostática apresentado no final da seção anterior.

Primeiro, vamos calcular o potencial $V_{\text {cav }}$ no interior da cavidade da Fig. 3. Trata-se, você sabe, da resolução da equação de Laplace, $\nabla^{2} V=0$, com as devidas condições de contorno. Vamos escolher um sistema de coordenadas em que o eixo $z$ tem a direção e o sentido do campo externo aplicado (veja as Figs. 2 e 3), e sua origem coincide com o centro da cavidade esférica. Mas este não é um problema simples, porque com $\gamma \neq 0$ (veja a Fig. 3 não temos simetria azimutal, e então a solução da equação de Laplace em coordenadas esféricas envolve os complicados harmônicos esféricos, usualmente explorados em disciplinas de eletrodinâmica clássica apenas ao nível de pós-graduação (veja, por exemplo, o capítulo 3 do livro do Jackson [6]). Mas existe uma forma criativa (e não menos rigorosa) de tornar a resolução deste problema mais simples, dispensando o uso de harmônicos esféricos. É o que veremos a seguir.

Inicialmente, vamos trabalhar com $\gamma=0$ (Fig.3). Temos, com $\gamma=0$, simetria azimutal, e então a forma geral da solução da equação de Laplace em coordenadas esféricas é

$$
V(r, \theta, \phi)=V(r, \theta)=\sum_{l=0}^{\infty}\left(A_{l} r^{l}+\frac{B_{l}}{r^{l+1}}\right) P_{l}(\cos \theta),
$$

em que as funções $P_{l}$ são os polinômios de Legendre $\left(P_{0}(x)=1, P_{1}(x)=x, P_{2}(x)=\left(3 x^{2}-1\right) / 2, \ldots\right)$. Temos, então,

$$
V_{\text {cav }}(r, \theta)=\sum_{l=0}^{\infty}\left(A_{l} r^{l}+\frac{B_{l}}{r^{l+1}}\right) P_{l}(\cos \theta) \quad(\text { para } r \leq a)
$$

e, fora da cavidade,

$$
V_{\text {fluido }}(r, \theta)=\sum_{l=0}^{\infty}\left(C_{l} r^{l}+\frac{D_{l}}{r^{l+1}}\right) P_{l}(\cos \theta) \quad(\text { para } r \geq a) .
$$


São quatro as condições de contorno para este problema. Uma delas,

$$
V_{\text {cav }}(a, \theta)=V_{\text {fluido }}(a, \theta),
$$

é a continuidade do potencial elétrico ao passarmos da cavidade para o meio contínuo ${ }^{10}$ Outra condição de contorno que também se aplica à transição da cavidade para o meio contínuo é

$$
\left.\epsilon_{0} \frac{\partial V_{\text {cav }}}{\partial r}\right|_{r=a}=\left.\epsilon \frac{\partial V_{\text {fluido }}}{\partial r}\right|_{r=a},
$$

que vem da condição mais geral $\epsilon_{\text {abaixo }}\left(\partial V_{\text {abaixo }} / \partial n\right)-$ $\epsilon_{\text {acima }}\left(\partial V_{\text {acima }} / \partial n\right)=\sigma_{f}$, em que $n$ denota uma coordenada normal à superfície de transição de um meio para o outro e $\sigma_{f}$ é a densidade superficial de carga livre nessa superfície (veja, por exemplo, a seção 4.4.2 (Boundary Value Problems with Linear Dielectrics) do livro do Griffiths [2]). A próxima condição de contorno diz respeito à aproximação esperada para $V_{\text {cav }}$ quando temos $r \ll a$ :

$$
V_{\text {cav }}(r, \theta) \approx V_{\text {dip }}(r, \theta)=\frac{1}{4 \pi \epsilon_{0}} \frac{p \cos \theta}{r^{2}}(\text { para } r \ll a) .
$$

Esta condição não é tão óbvia quanto possa parecer à primeira vista. Perceba que ao expressá-la estamos considerando um dipolo puro no centro da cavidade, o que é algo um tanto estranho para uma (já estranha) cavidade microscópica, não acha? Mas faz parte da modelagem. A última condição de contorno diz respeito à aproximação esperada para $V_{\text {fluido }}$ quando temos $r \gg a$ :

$$
V_{\text {fluido }}(r, \theta) \approx \underbrace{-E r \cos \theta}_{-E z}+\text { constante }(\text { para } r \gg a) \text {. }
$$

E por que essa aproximação é esperada? Porque ela nos dá, para $r \gg a, \boldsymbol{E}=-\boldsymbol{\nabla} V_{\text {fluido }} \approx E \hat{z}$, que é o campo macroscópico uniforme no fluido antes da introdução da cavidade microscópica (veja a Fig.2). Longe da cavidade (ou seja, com $r \gg a$ ), esse campo permanece praticamente o mesmo.

Aplicando essas quatro condições de contorno às expressões gerais acima para $V_{\text {cav }}(r, \theta)$ e/ou $V_{\text {fluido }}(r, \theta)$, obtemos 11

$$
V_{\text {cav }}(r, \theta)=\underbrace{\frac{1}{4 \pi \epsilon_{0}} \frac{p \cos \theta}{r^{2}}}_{V_{\text {dip }}(r, \theta)}+A_{1} \underbrace{r \cos \theta}_{z},
$$

com

$$
A_{1}=\left(\frac{-3 \epsilon}{2 \epsilon+\epsilon_{0}}\right) E-\left(\frac{\epsilon-\epsilon_{0}}{2 \epsilon+\epsilon_{0}}\right) \frac{p}{2 \pi \epsilon_{0} a^{3}} .
$$


acima quando afirmamos que as expressões para $V_{\text {cav }}(r, \theta)$ e para $V_{\text {fluido }}(r, \theta)$ valem, ambas, para $r=a$.

${ }^{11} \mathrm{O}$ nível de dificuldade aqui corresponde ao de um problema típico de uma lista de exercícios de uma disciplina de graduação de eletrodinâmica clássica.
}

Pois bem, uma vez calculado $V_{\text {cav }}$, temos

$$
\begin{aligned}
V_{\mathrm{int}} & =V_{\mathrm{cav}}-V_{\mathrm{dip}} \\
& =\left[\left(\frac{-3 \epsilon}{2 \epsilon+\epsilon_{0}}\right) E-\left(\frac{\epsilon-\epsilon_{0}}{2 \epsilon+\epsilon_{0}}\right) \frac{p}{2 \pi \epsilon_{0} a^{3}}\right] z,
\end{aligned}
$$

e então obtemos, a partir de $\boldsymbol{E}_{\text {int }}=-\nabla V_{\text {int }}$ (observando que $E \hat{z}=\boldsymbol{E}$ e $p \hat{z}=\boldsymbol{p}$, e optando por trabalhar com $\left.\epsilon_{r} \equiv \epsilon / \epsilon_{0}\right)$,

$$
\boldsymbol{E}_{\mathrm{int}}=\left(\frac{3 \epsilon_{r}}{2 \epsilon_{r}+1}\right) \boldsymbol{E}+\left(\frac{\epsilon_{r}-1}{2 \epsilon_{r}+1}\right) \frac{\boldsymbol{p}}{2 \pi \epsilon_{0} a^{3}} .
$$

Você sabe, esta expressão foi obtida para o caso particular em que $\gamma=0$ (Fig. 3). Contudo, veremos que ela continua válida para $\gamma \neq 0$ (e, portanto, $\operatorname{com} \boldsymbol{p}$ não necessariamente paralelo a $\boldsymbol{E})$. Ou seja, esta já é a expressão para o campo interno do modelo de Onsager!

Quando $p$ tende a 0 (ou seja, eliminando o dipolo da cavidade), o campo $\boldsymbol{E}_{\text {int }}$ tende a

$$
\boldsymbol{G} \equiv\left(\frac{3 \epsilon_{r}}{2 \epsilon_{r}+1}\right) \boldsymbol{E} .
$$

Este campo (uniforme) é propriamente denominado campo de cavidade. Agora, quando $E$ tende a 0 (o que corresponde a "desligar" o campo externo $\boldsymbol{E}_{\text {ext }}$ aplicado ao fluido) o campo $\boldsymbol{E}_{\text {int }}$ tende a

$$
\boldsymbol{R} \equiv\left(\frac{\epsilon_{r}-1}{2 \epsilon_{r}+1}\right) \frac{\boldsymbol{p}}{2 \pi \epsilon_{0} a^{3}} .
$$

Este campo (também uniforme) é propriamente denominado campo de reação, porque é o campo que age sobre o dipolo na cavidade em resposta (ou "reação") à polarização induzida por ele no fluido. O campo $\boldsymbol{E}_{\text {int }}$ em (14), obtido para o caso particular em que $\gamma=0$, é então a soma dos campos $\boldsymbol{G}$ e $\boldsymbol{R}$. Tenha em mente que $\boldsymbol{G}$ é o campo na cavidade sem o dipolo, com o campo externo ligado, enquanto $\boldsymbol{R}$ é o campo que age sobre o dipolo na cavidade, com o campo externo desligado. É claro, as expressões para os campos $\boldsymbol{G}$ e $\boldsymbol{R}$ permanecem as mesmas no caso em que $\gamma \neq 0$ (Fig. 3 ), porque esses campos podem ser calculados independentemente um do outro. Cada um deles é a solução de um problema de eletrostática distinto, com simetria azimutal, e esses problemas constituem dois casos particulares do problema que acabamos de resolver. A "sacada" é que, devido ao princípio da superposição e à linearidade do dielétrico, ainda podemos, com $\gamma \neq 0$, expressar o campo $\boldsymbol{E}_{\text {int }}$ como a soma dos campos $\boldsymbol{G}$ e $\boldsymbol{R}$. (Pense um pouco sobre isso fisicamente, em vez de matematicamente. Se essa combinação dos campos $\boldsymbol{G}$ e $\boldsymbol{R}$ vale para $\gamma=0$, por que não valeria para $\gamma \neq 0$, dada a linearidade do dielétrico?) Portanto, o campo interno do modelo de Onsager é

$$
\boldsymbol{E}_{\mathrm{int}}=\boldsymbol{G}+\boldsymbol{R}
$$

com $\boldsymbol{G}$ e $\boldsymbol{R}$ dados respectivamente pelas igualdades 15 e (16), mas com $\boldsymbol{p}$ não necessariamente paralelo a $\boldsymbol{E}$ (veja as Figs. 4 e 5 ). 


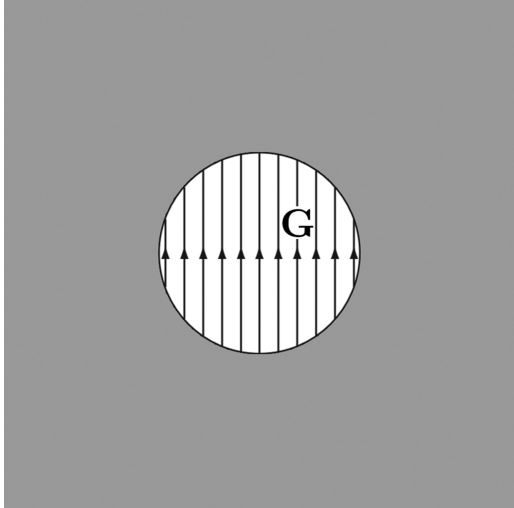

Figura 4: Campo de cavidade $\boldsymbol{G}$.



Figura 5: Campo de reação $\boldsymbol{R}$, que tem a direção e o sentido do dipolo puro no centro da cavidade (não ilustrado na figura). As linhas de campo além da cavidade correspondem ao campo macroscópico no dielétrico (com o campo externo desligado).

Se você (sem razão) torceu o nariz para o argumento acima, pode tentar calcular o campo interno do modelo de Onsager considerando, desde o início, $\gamma \neq 0$ (e então lidando com harmônicos esféricos). Mas, podemos garantir (acredite, fizemos as contas pra você), a expressão que encontrará para $\boldsymbol{E}_{\text {int }}$ será a mesma presente em (14.

Antes de nos encaminharmos para o passo 2, vamos analisar os módulos dos campos $\boldsymbol{G}$ e $\boldsymbol{R}$, e então comparálos.

Como $\epsilon_{r}>1$, a partir da igualdade 15 podemos concluir que o módulo de $\boldsymbol{G}$ é maior que o módulo de $\boldsymbol{E}$ (o campo macroscópico uniforme longe da cavidade). Isto é esperado (nesta modelagem), devido ao acúmulo de carga positiva na parte inferior da cavidade e ao acúmulo de carga negativa em sua parte superior (visualize a distribuição de dipolos puros). Quando $\epsilon_{r} \rightarrow 1^{+}, \boldsymbol{G}$ tende a $\boldsymbol{E}$ (ou, seria mais correto dizer, $\boldsymbol{G}$ e $\boldsymbol{E}$ tendem ambos a $\boldsymbol{E}_{\text {ext }}$ ), e quando $\epsilon_{r} \rightarrow \infty, \boldsymbol{G}$ tende a apenas $3 \boldsymbol{E} / 2$ (ou, mais corretamente, a diferença entre $3 \boldsymbol{E} / 2$ e $\boldsymbol{G}$ tende a 0 , sendo que o próprio campo $\boldsymbol{E}$ tende a 0 - embora mais lentamente que a diferença acima) 12

\footnotetext{
$12 \overline{\text { Lembre-se que } \boldsymbol{E}=\boldsymbol{E}_{\text {ext }}} / \epsilon_{r}$. Assim, mesmo com um valor de $\epsilon_{r}$ elevado, podemos manter para $\boldsymbol{E}$ uma intensidade apreciável com um campo externo suficientemente intenso (se o dielétrico ainda se comportar de forma linear). Você pode verificar que
}

Para $\epsilon_{r}=80$ (o valor aproximado da constante dielétrica da água pura, a $20^{\circ} \mathrm{C}$, que é bastante elevado), temos $\boldsymbol{G} \approx 1,49 \boldsymbol{E}$. Mas a razão entre os módulos de $\boldsymbol{G}$ e $\boldsymbol{E}$ já é igual a 1,45 para $\epsilon_{r}=14,5$.

O módulo de $\boldsymbol{R}$ depende não só de $\epsilon_{r}$, mas também do raio $a$ da cavidade e do módulo de $\boldsymbol{p}$, e este módulo muda constantemente (veja a igualdade (21), logo adiante). Então é interessante calcularmos o módulo do campo de reação médio,

$$
\overline{\boldsymbol{R}}=\left(\frac{\epsilon_{r}-1}{2 \epsilon_{r}+1}\right) \frac{\overline{\boldsymbol{p}}}{2 \pi \epsilon_{0} a^{3}}
$$

Podemos escrever (volte à seção 2, se necessário):

$$
\boldsymbol{P}=N \overline{\boldsymbol{p}}=\frac{1}{4 \pi a^{3} / 3} \overline{\boldsymbol{p}}=\frac{\overline{\boldsymbol{p}}}{4 \pi a^{3} / 3},
$$

em que, obviamente, $4 \pi a^{3} / 3$ é o volume da cavidade esférica ${ }^{13}$ Como $\boldsymbol{P}=\epsilon_{0} \chi_{e} \boldsymbol{E}=\epsilon_{0}\left(\epsilon_{r}-1\right) \boldsymbol{E}$, obtemos:

$$
\overline{\boldsymbol{p}}=\frac{4}{3} \pi a^{3} \epsilon_{0}\left(\epsilon_{r}-1\right) \boldsymbol{E} .
$$

Segue então que

$$
\overline{\boldsymbol{R}}=\frac{2}{3} \frac{\left(\epsilon_{r}-1\right)^{2}}{2 \epsilon_{r}+1} \boldsymbol{E} .
$$

Observe que no caso particular em que $\boldsymbol{E}_{\text {ext }}=0$ temos $\boldsymbol{E}=0$ e, portanto, $\overline{\boldsymbol{p}}=0, \boldsymbol{P}=0$ e $\overline{\boldsymbol{R}}=0$, como esperado. Para $\epsilon_{r}=80$, temos $\overline{\boldsymbol{R}} \approx 26 \boldsymbol{E}$, e, assim, $|\overline{\boldsymbol{R}}| \approx 17|\boldsymbol{G}|$. Mas para $\epsilon_{r} \approx 2,3$ (o valor aproximado da constante dielétrica do benzeno líquido, a $20^{\circ} \mathrm{C}$ ), temos $|\overline{\boldsymbol{R}}| \approx 0,16|\boldsymbol{G}|$. Você pode verificar que $|\overline{\boldsymbol{R}}|=|\boldsymbol{G}|$ para $\epsilon_{r} \approx 6,34$. Você esperava que o campo de reação pudesse atingir valores tão elevados, em comparação com o campo de cavidade?

\section{Passo 2}

Este passo é curto. Substituindo (17) (ou, equivalentemente, (14) ) em (11), e resolvendo a equação resultante para $\boldsymbol{p}$, obtemos:

$$
\boldsymbol{p}=\frac{\boldsymbol{p}_{0}+\left(\frac{3 \epsilon_{r}}{2 \epsilon_{r}+1}\right) \alpha \boldsymbol{E}}{1-\left(\frac{\epsilon_{r}-1}{2 \epsilon_{r}+1}\right) \frac{\alpha}{2 \pi \epsilon_{0} a^{3}}} .
$$

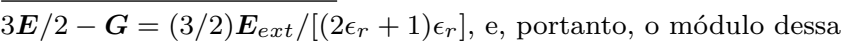
diferença escala com $1 / \epsilon_{r}^{2}$ quando $\epsilon_{r} \gg 1$, então tendendo a 0 muito mais rapidamente que o módulo de $\boldsymbol{E}$, quando $\epsilon_{r} \rightarrow \infty$.

${ }^{13}$ Seja $\mathcal{N}$ o número de moléculas em um volume macroscópico $\mathscr{V}$ do fluido. Em seu artigo, Onsager [1] usou a aproximação $\mathscr{V} / \mathcal{N} \approx$ $4 \pi a^{3} / 3$, de onde vem a relação $N=\mathscr{N} / \mathscr{V} \approx 1 /\left(4 \pi a^{3} / 3\right)$. Trata-se de uma aproximação porque, é claro, o volume do fluido não pode ser totalmente preenchido por volumes esféricos. Lembre-se que $a$ é o raio de uma cavidade esférica microscópica imaginária onde se encontra uma molécula do fluido (veja a Fig. 33), sendo da ordem do tamanho da molécula apenas para líquidos. Assim, o valor de $a$ no modelo de Onsager é determinado pela distância média entre as moléculas do fluido, não pelas dimensões moleculares, e por isso quando o valor de $a$ aumenta, a densidade numérica $N$ diminui. 


\section{Passo 3}

Tomando a média de ambos os membros da igualdade (21) obtemos

$$
\overline{\boldsymbol{p}}=\frac{\overline{\boldsymbol{p}}_{\mathbf{0}}+\left(\frac{3 \epsilon_{r}}{2 \epsilon_{r}+1}\right) \alpha \boldsymbol{E}}{1-\left(\frac{\epsilon_{r}-1}{2 \epsilon_{r}+1}\right) \frac{\alpha}{2 \pi \epsilon_{0} a^{3}}},
$$

já que o campo macroscópico $\boldsymbol{E}$ é constante, e, portanto, $\overline{\boldsymbol{E}}=\boldsymbol{E}$. Assim, obteremos uma expressão para $\overline{\boldsymbol{p}}$ calculando $\bar{p}_{0}$ e substituindo em $(22)$ a expressão encontrada.

Vimos na seção 2 que $\overline{\boldsymbol{p}}=\left(\sum_{r} \boldsymbol{p}_{r} e^{-\beta \varepsilon_{r}}\right) /\left(\sum_{r} e^{-\beta \varepsilon_{r}}\right)$, em que $\boldsymbol{p}_{r}$ é o momento de dipolo total da molécula quando ela está no estado $r$, de energia $\varepsilon_{r}$. Esta é a forma como calculamos médias no ensemble canônico, e ela se aplica a qualquer variável do sistema, não apenas a $\boldsymbol{p}$. Assim, podemos substituir $\boldsymbol{p}$ por $\boldsymbol{p}_{0}$ (mantendo as outras grandezas na expressão), obtendo

$$
\overline{\boldsymbol{p}}_{\mathbf{0}}=\frac{\sum_{r}\left(\boldsymbol{p}_{0}\right)_{r} e^{-\beta \varepsilon_{r}}}{\sum_{r} e^{-\beta \varepsilon_{r}}} .
$$

Se você cursou uma disciplina de mecânica estatística em sua graduação, praticou o tipo de fatoração que faremos a seguir. Podemos separar $\varepsilon_{r}$ nos termos de energia cinética, $t_{r}$, e energia potencial, $u_{r}$, e com isso obtemos

$$
e^{-\beta \varepsilon_{r}}=e^{-\beta\left(t_{r}+u_{r}\right)}=e^{-\beta t_{r}} e^{-\beta u_{r}} .
$$

O estado $r$ é determinado por coordenadas generalizadas $q_{1}, q_{2}, \ldots$ e seus momentos associados $p_{1}, p_{2}, \ldots$ (não precisamos nos preocupar com sua especificação agora), $\operatorname{mas} t_{r}=t_{p_{1}, p_{2}, \ldots} \equiv t_{p}, u_{r}=u_{q_{1}, q_{2}, \ldots} \equiv u_{q}$ e $\left(\boldsymbol{p}_{0}\right)_{r}=$ $\left(\boldsymbol{p}_{0}\right)_{q_{1}, q_{2}, \ldots} \equiv\left(\boldsymbol{p}_{0}\right)_{q}$, de modo que podemos reescrever a igualdade 23 como

$$
\begin{aligned}
\overline{\boldsymbol{p}}_{\mathbf{0}} & =\frac{\sum_{p} \sum_{q}\left(\boldsymbol{p}_{0}\right)_{q} e^{-\beta t_{p}} e^{-\beta u_{q}}}{\sum_{p} \sum_{q} e^{-\beta t_{p}} e^{-\beta u_{q}}} \\
& =\frac{\left(\sum_{p} e^{-\beta t_{p}}\right)\left(\sum_{q}\left(\boldsymbol{p}_{0}\right)_{q} e^{-\beta u_{q}}\right)}{\left(\sum_{p} e^{-\beta t_{p}}\right)\left(\sum_{q} e^{-\beta u_{q}}\right)} .
\end{aligned}
$$

Agora, vamos especificar as coordenadas generalizadas $q_{1}, q_{2}, \ldots$ para este problema. Você sabe, a molécula na cavidade esférica microscópica está sendo modelada como um dipolo, com momento de dipolo permanente $\boldsymbol{p}_{0}$, momento de dipolo induzido $\alpha \boldsymbol{E}_{\text {int }}$ e momento de dipolo total $\boldsymbol{p}=\boldsymbol{p}_{0}+\alpha \boldsymbol{E}_{\text {int }}$. Esta igualdade já mostra que esses três vetores não são independentes. Mas, além disso, lembre-se que a expressão para $\boldsymbol{E}_{\text {int }}$, em (14), envolve o momento de dipolo total $\boldsymbol{p}$. Portanto, os três vetores estão relacionados ${ }^{14}$ e então podemos usar $\boldsymbol{p}$ ou $\boldsymbol{p}_{0}$ para especificar a configuração da molécula na cavidade. A escolha natural é $\boldsymbol{p}_{0}$, porque esse vetor sempre acompanha, rotacionalmente, a molécula considerada (visualize

\footnotetext{
${ }^{14}$ Essa relação fica clara observando-se que a igualdade 21 nos mostra que $\boldsymbol{p}_{0}$ determina $\boldsymbol{p}$, e então, através da igualdade $[14, \boldsymbol{p}$ determina $\boldsymbol{E}_{\text {int }}$.
}

isso para a molécula de água). Em outras palavras, em um sistema de coordenadas interno à molécula, $\boldsymbol{p}_{0}$ é um vetor constante. Assim, usando o vetor $\boldsymbol{p}_{0}$ para especificar a configuração da molécula, podemos reescrever a igualdade 24 como

$$
\overline{\boldsymbol{p}}_{\mathbf{0}}=\frac{\sum_{\boldsymbol{p}_{0}} \boldsymbol{p}_{0} e^{-\beta u_{\boldsymbol{p}_{0}}}}{\sum_{\boldsymbol{p}_{0}} e^{-\beta u_{\boldsymbol{p}_{0}}}}
$$

significando que as somas são realizadas sobre todos as possíveis orientações para $\boldsymbol{p}_{0}$ (já que seu módulo não muda). É claro, essas somas devem ser substituídas por integrais nas variáveis $\theta$ e $\phi$, que especificam, em coordenadas esféricas, a direção de $\boldsymbol{p}_{0}$. Faremos isso logo adiante, mas, antes, vamos obter uma expressão para $u_{p_{0}}$

A obtenção de uma expressão para $u_{\boldsymbol{p}_{0}}$ envolve uma sutileza. Se você cursou uma disciplina de eletrodinâmica (ou eletromagnetismo) em sua graduação, certamente aprendeu que a energia potencial de um dipolo, com momento de dipolo $\boldsymbol{p}$, submetido a um campo elétrico $\boldsymbol{E}$, pode ser expressa como $U=-\boldsymbol{p} \cdot \boldsymbol{E}$. Mas provavelmente a dedução que lhe foi apresentada para esta expressão foi feita considerando-se um dipolo rígido - ou seja, que não possui momento de dipolo induzido, só o momento de dipolo permanente. Não é o caso, aqui. Então precisamos reobter a expressão para essa energia potencial, mas agora considerando que $\boldsymbol{p}=\boldsymbol{p}_{0}+\alpha \boldsymbol{E}_{\text {int }}$. Em seu artigo [1], Onsager obteve uma expressão para a energia potencial de interação do dipolo na cavidade com o campo interno partindo do cálculo do torque $\boldsymbol{\Gamma}$ que esse campo produz no dipolo:

$$
\Gamma=p \times E_{\text {int }} .
$$

Substituindo 11 em 26, obtemos:

$$
\boldsymbol{\Gamma}=\left(\boldsymbol{p}_{0}+\alpha \boldsymbol{E}_{\mathrm{int}}\right) \times \boldsymbol{E}_{\mathrm{int}}=\boldsymbol{p}_{0} \times \boldsymbol{E}_{\mathrm{int}} .
$$

Como $\boldsymbol{p}_{0} \times \boldsymbol{p}_{0}=0$, é claramente interessante explicitarmos $\boldsymbol{p}_{0}$ na expressão que usaremos para $\boldsymbol{E}_{\text {int }}$ em (27). Para isso, vamos substituir (21) em 14, e então a expressão resultante em (27). Após algumas manipulações algébricas, obtemos:

$$
\boldsymbol{\Gamma}=\left(\frac{3 \epsilon_{r}}{2 \epsilon_{r}+1-\left(\epsilon_{r}-1\right) \alpha /\left(2 \pi \epsilon_{0} a^{3}\right)}\right) \boldsymbol{p}_{0} \times \boldsymbol{E} .
$$

Este resultado é muito interessante, porque a expressão entre parênteses, que denotaremos por $\xi$, é constante

\footnotetext{
${ }^{15} \mathrm{Um}$ aparte: Observe que a distinção entre $\boldsymbol{p}$ e $\boldsymbol{p}_{0}$ é mais matemática que física. Sabemos que $\boldsymbol{p}_{0}$ é o momento de dipolo da molécula (em uma certa orientação) na ausência de campo elétrico aplicado sobre ela. Mas se um campo $\boldsymbol{E}_{\text {int }}$ atua sobre a molécula em um determinado instante, assim promovendo uma separação adicional de cargas, o momento de dipolo $\boldsymbol{p}_{0}$ inicial (antes da aplicação do campo $\boldsymbol{E}_{\text {int }}$ ) "se perde", entende? Ou seja, simplesmente não existe mais a distribuição de cargas que resulta em $\boldsymbol{p}_{0}$, mas, matematicamente, podemos expressar o novo momento de dipolo como $\boldsymbol{p}=\boldsymbol{p}_{0}+\alpha \boldsymbol{E}_{\text {int }}$. Entendendo isso, você pode perguntar: então faz algum sentido calcularmos $\overline{\boldsymbol{p}}_{\mathbf{0}}$ ? A resposta é que faz sentido, sim, porque podemos pensar $\boldsymbol{p}_{0}$ agora como $\boldsymbol{p}_{0} \equiv \boldsymbol{p}-\alpha \boldsymbol{E}_{\text {int }}$. Este vetor tem módulo constante, e sempre acompanha, rotacionalmente, a molécula.
} 
(ou seja, não muda à medida que molécula rotaciona na cavidade), e, assim, podemos pensar (matematicamente) o torque em (28) como o torque exercido por um campo constante $\boldsymbol{E}$ sobre um dipolo rígido de momento de dipolo $\xi \boldsymbol{p}_{0}$. O resultado é que podemos usar, para a energia potencial $u_{\boldsymbol{p}_{0}}$, que é a energia de interação do dipolo na cavidade (de momento de dipolo $\boldsymbol{p}$ ) com o campo $\boldsymbol{E}_{\text {int }}$, a expressão clássica $-\xi \boldsymbol{p}_{0} \cdot \boldsymbol{E}$. Bacana, não é? Temos, então:

$$
u_{\boldsymbol{p}_{0}}=-\xi \boldsymbol{p}_{0} \cdot \boldsymbol{E}
$$

com

$$
\xi \equiv \frac{3 \epsilon_{r}}{2 \epsilon_{r}+1-\left(\epsilon_{r}-1\right) \alpha /\left(2 \pi \epsilon_{0} a^{3}\right)} .
$$

Substituindo 29 em 25 obtemos:

$$
\overline{\boldsymbol{p}}_{\mathbf{0}}=\frac{\sum_{\boldsymbol{p}_{0}} \boldsymbol{p}_{0} e^{\beta \xi \boldsymbol{p}_{0} \cdot \boldsymbol{E}}}{\sum_{\boldsymbol{p}_{0}} e^{\beta \xi \boldsymbol{p}_{0} \cdot \boldsymbol{E}}} .
$$

Agora sim, vamos substituir essas somas por integrais nas variáveis $\theta$ e $\phi$ que especificam a direção de $\boldsymbol{p}_{0}$. Mantendo a escolha feita no início desta seção, o nosso sistema de coordenadas tem o eixo $z$ com a mesma direção e o mesmo sentido que o campo $\boldsymbol{E}$, e assim $\boldsymbol{p}_{0} \cdot \boldsymbol{E}=p_{0} E \cos \theta$. Perceba que o vetor $\boldsymbol{p}_{0}$ deve varrer uma superfície esférica (da raio arbitrário $b$ ), cujo elemento de área em coordenadas esféricas é $b^{2} \operatorname{sen} \theta \mathrm{d} \theta \mathrm{d} \phi$. Temos, então:

$$
\overline{\boldsymbol{p}}_{\mathbf{0}}=\frac{\int_{0}^{\pi} \int_{0}^{2 \pi} \boldsymbol{p}_{0} e^{\beta \xi p_{0} E \cos \theta} \partial^{2} \operatorname{sen} \theta \mathrm{d} \theta \mathrm{d} \phi}{\int_{0}^{\pi} \int_{0}^{2 \pi} e^{\beta \xi p_{0} E \cos \theta} \partial^{2} \operatorname{sen} \theta \mathrm{d} \theta \mathrm{d} \phi},
$$

com

$$
\begin{aligned}
& \boldsymbol{p}_{0}=p_{0 x} \hat{\boldsymbol{x}}+p_{0 y} \hat{\boldsymbol{y}}+p_{0 z} \hat{\boldsymbol{z}}, \\
& p_{0 x}=p_{0} \operatorname{sen} \theta \cos \phi, \\
& p_{0 y}=p_{0} \operatorname{sen} \theta \operatorname{sen} \phi, \\
& p_{0 z}=p_{0} \cos \theta .
\end{aligned}
$$

Mas sabemos que, por simetria, $\bar{p}_{\mathbf{0}}$ tem a direção e o sentido do campo $\boldsymbol{E}$ - ou seja, $\overline{\boldsymbol{p}}_{\mathbf{0}}=\bar{p}_{0_{z}} \hat{\boldsymbol{z}}$ (que é o mesmo que dizer que $\left.\overline{p_{0 x}}=\bar{p}_{0 y}=0\right)$. Assim, podemos reescrever a igualdade (31) diretamente como

$$
\overline{\boldsymbol{p}}_{\mathbf{0}}=p_{0} \hat{\boldsymbol{z}} \frac{\int_{0}^{\pi} \int_{0}^{2 \pi} \cos \theta e^{\beta \xi p_{0} E \cos \theta} \operatorname{sen} \theta \mathrm{d} \theta \mathrm{d} \phi}{\int_{0}^{\pi} \int_{0}^{2 \pi} e^{\beta \xi p_{0} E \cos \theta} \operatorname{sen} \theta \mathrm{d} \theta \mathrm{d} \phi} .
$$

Desenvolvendo, obtemos:

$$
\begin{aligned}
\overline{\boldsymbol{p}}_{\mathbf{0}} & =p_{0} \hat{\boldsymbol{z}} \frac{\left(\int_{0}^{2 \pi} \mathrm{d} \phi\right) \int_{0}^{\pi} \cos \theta e^{\beta \xi p_{0} E \cos \theta} \operatorname{sen} \theta \mathrm{d} \theta}{\left(\int_{0}^{2 \pi}(\phi) \int_{0}^{\pi} e^{\beta \xi p_{0} E \cos \theta} \operatorname{sen} \theta \mathrm{d} \theta\right.} \\
= & p_{0} \hat{\boldsymbol{z}} \frac{\int_{0}^{\pi} \cos \theta e^{\beta \xi p_{0} E \cos \theta} \operatorname{sen} \theta \mathrm{d} \theta}{\int_{0}^{\pi} e^{\beta \xi p_{0} E \cos \theta} \operatorname{sen} \theta \mathrm{d} \theta} .
\end{aligned}
$$

E usando um "truque matemático" muito conhecido na mecânica estatística, podemos reescrever:

$$
\overline{\boldsymbol{p}}_{\mathbf{0}}=p_{0} \hat{\boldsymbol{z}} \frac{\partial}{\partial v} \ln \left(\int_{0}^{\pi} e^{v \cos \theta} \operatorname{sen} \theta \mathrm{d} \theta\right), \quad v \equiv \beta \xi p_{0} E .
$$

As contas que seguem são simples, e o resultado é (lembrando que $\beta=1 / k T)$ :

$$
\overline{\boldsymbol{p}}_{\mathbf{0}}=p_{0} \hat{\boldsymbol{z}}\left(\operatorname{coth}\left(\xi p_{0} E / k T\right)-\frac{1}{\xi p_{0} E / k T}\right),
$$

com $\xi$ definido em 30 .

Temos, na expressão entre parênteses em 32 , a chamada função de Langevin: $L(x) \equiv \operatorname{coth}(x)-1 / x$. Onsager [1] considerou apenas campos elétricos de baixa intensidade ${ }^{16}$ no sentido de que

$$
x \equiv \xi p_{0} E / k T \ll 1,
$$

e então fez uso da aproximação

$$
L(x) \approx \frac{x}{3}, \text { para }|x| \ll 1 .
$$

Com essa aproximação (cuja dedução disponibilizamos online como Material Suplementar), podemos reescrever a igualdade 32 como

$$
\overline{\boldsymbol{p}}_{\mathbf{0}} \approx \xi \frac{p_{0}^{2} E}{3 k T} \hat{\boldsymbol{z}}=\xi \frac{p_{0}^{2}}{3 k T} \boldsymbol{E} .
$$

Substituindo 35 em (22) (fazendo uso da expressão para $\xi$ em (30), e então (22) em (12), obtemos:

$$
\begin{aligned}
\boldsymbol{P} & =\left(\frac{3 \epsilon_{r}}{2 \epsilon_{r}+1-\left(\epsilon_{r}-1\right) \alpha /\left(2 \pi \epsilon_{0} a^{3}\right)} \frac{p_{0}^{2}}{3 k T}+\frac{3 \epsilon_{r} \alpha}{2 \epsilon_{r}+1}\right) \\
& \times\left[1-\left(\frac{\epsilon_{r}-1}{2 \epsilon_{r}+1}\right) \frac{\alpha}{2 \pi \epsilon_{0} a^{3}}\right]^{-1} N \boldsymbol{E} .
\end{aligned}
$$

\section{Passo 4}

Agora, tomando o módulo de $\boldsymbol{P}$ em 36 , e substituindo-o em (13), obtemos:

$$
\begin{array}{r}
\frac{\epsilon_{0}\left(\epsilon_{r}-1\right)}{N}\left[1-\left(\frac{\epsilon_{r}-1}{2 \epsilon_{r}+1}\right) \frac{\alpha}{2 \pi \epsilon_{0} a^{3}}\right]= \\
\left(\frac{3 \epsilon_{r}}{2 \epsilon_{r}+1-\left(\epsilon_{r}-1\right) \alpha /\left(2 \pi \epsilon_{0} a^{3}\right)}\right) \frac{p_{0}^{2}}{3 k T}+\frac{3 \epsilon_{r} \alpha}{2 \epsilon_{r}+1} .
\end{array}
$$

Podemos "nos livrar" do raio a da cavidade esférica microscópica, na equação acima, usando a relação (veja a nota de rodapé 13$) N=1 /\left(4 \pi a^{3} / 3\right)$. Após algumas manipulações algébricas, obtemos:

$$
\begin{aligned}
\frac{\epsilon_{r}-1}{\epsilon_{r}+2} & =\frac{N \alpha}{3 \epsilon_{0}} \\
& +\left(\frac{9 \epsilon_{r} /\left(\epsilon_{r}+2\right)}{2 \epsilon_{r}+1-2\left(\epsilon_{r}-1\right) N \alpha / 3 \epsilon_{0}}\right) \frac{N}{3 \epsilon_{0}} \frac{p_{0}^{2}}{3 k T} .
\end{aligned}
$$

${ }^{16}$ Lembre-se que estamos supondo que o fluido dielétrico é linear, e isso não depende apenas do meio, mas também da intensidade do campo, que deve ser suficientemente baixa. 
Esta seria a equação de Onsager (a menos de uma possível reorganização da mesma), se ele tivesse optado por manter a polarizabilidade $\alpha$ em sua equação final. Mas Onsager escolheu trabalhar com o índice de refração $n$ do fluido, em vez de $\alpha$. Vamos explicar como isso pode ser feito.

O índice de refração $n$ de um meio é definido como a razão entre a velocidade da luz no vácuo $(c)$ e a velocidade $v$ da luz nesse meio; ou seja, $n \equiv c / v$. De acordo com a eletrodinâmica clássica, $c=1 / \sqrt{\mu_{0} \epsilon_{0}}$, e, para meios lineares, $v=1 / \sqrt{\mu \epsilon}$ (veja, por exemplo, as seções 9.2 .1 e 9.3.1 do Griffiths [2]). Como, para a maioria dos materiais, a permeabilidade magnética, $\mu$, é muito próxima da permeabilidade magnética do vácuo, $\mu_{0}$ (veja, por exemplo, a Tabela 6.1 do Griffiths 2]), temos, para esses materiais, $n=\sqrt{\epsilon / \epsilon_{0}}=\sqrt{\epsilon_{r}}$, ou, equivalentemente, $\epsilon_{r}=n^{2}$. Mas atenção: a constante dielétrica $\epsilon_{r}$ é uma constante no sentido de que seu valor não depende da intensidade do campo elétrico macroscópico $\boldsymbol{E}$ (desde que, é claro, tal intensidade seja suficientemente baixa para que o meio possa ser classificado como linear). Contudo, seu valor depende da frequência do campo elétrico $\boldsymbol{E}$ ! E é por isso que a constante dielétrica $\epsilon_{r}$ com que trabalhamos até a igualdade (38), e que aparece na equação final de Onsager (que obteremos logo adiante), é denominada constante dielétrica estática: ela se aplica ao caso particular de campos elétricos estáticos (ou praticamente estáticos). Na relação $\epsilon_{r}=n^{2}$, temos $\epsilon_{r}=\epsilon_{r}(\nu)$, em que $\nu$ é a frequência de $\boldsymbol{E}$ (tipicamente uma frequência óptica), enquanto na igualdade (38) temos o caso particular $\epsilon_{r}=\epsilon_{r}(0)$. Por exemplo, para a água pura a $20^{\circ} \mathrm{C}$, submetida a luz visível, temos $n \approx 1,33$, o que nos dá $\epsilon_{r} \approx 1,77$ - que é um valor bem diferente de 80 (a constante dielétrica estática da água pura, a $20^{\circ} \mathrm{C}$ ), não é? Portanto, a relação $\epsilon_{r}=n^{2}$ não pode ser usada na igualdade (38). No entanto, quando um meio dielétrico linear é submetido a um campo elétrico que oscila com frequência óptica, os momentos de dipolo permanentes não acompanham as oscilações do campo, e, assim, é como se eles não existissem! Isso porque as orientações moleculares ficam com uma distribuição aleatória, e então os momentos de dipolo permanentes não contribuem para a polarização do meio. Nesses casos, só o momento de dipolo induzido contribui para o valor da constante dielétrica - e por isso o valor de $\epsilon_{r}$ em frequências ópticas pode ser tão menor que seu valor para campos estáticos. Podemos então fazer $\epsilon_{r}=n^{2}$ em (38), desde que façamos também $p_{0}=0$, e daí obtemos a seguinte relação:

$$
\frac{n^{2}-1}{n^{2}+2}=\frac{N \alpha}{3 \epsilon_{0}} \text {. }
$$

Substituindo $N \alpha / 3 \epsilon_{0}$, em 38 , por $\left(n^{2}-1\right) /\left(n^{2}+2\right)$, e reorganizando a equação resultante, obtemos

$$
\frac{\left(\epsilon_{r}-n^{2}\right)\left(2 \epsilon_{r}+n^{2}\right)}{\epsilon_{r}\left(n^{2}+2\right)^{2}}=\frac{N}{3 \epsilon_{0}} \frac{p_{0}^{2}}{3 k T},
$$

que é a equação de Onsager. Esperamos ter ficado absolutamente claro que não se trata de uma relação exata entre as grandezas $\epsilon_{r}, n, N, p_{0}$ e $T$, para um fluido polar puro, mas de uma relação obtida por um processo de modelagem, através de uma série de considerações e simplificações que devem ser observadas por quem faz uso da mesma. Por exemplo, de forma alguma a equação de Onsager se aplica a moléculas longas, ou a meios não lineares. E mesmo para meios lineares constituídos de moléculas com geometria não muito distinta de uma geometria esférica, aproximações muito fortes foram realizadas, como considerar a vizinhança imediata de uma determinada molécula do fluido como constituindo um meio contínuo. Pode haver certas correlações entre as orientações de moléculas vizinhas que o modelo de Onsager simplesmente ignora. É o caso, por exemplo, para água pura, onde moléculas vizinhas interagem através das chamadas ligações de hidrogênio. O valor fornecido pela equação de Onsager para o módulo do momento de dipolo permanente da água, considerando-se água pura a $20^{\circ} \mathrm{C}(T=293 \mathrm{~K})$, para a qual temos $n \approx 1,33$, $\epsilon_{r} \approx 80 \mathrm{e} N \approx 3,34 \times 10^{28} \mathrm{~m}^{-3}$, é $p_{0} \approx 10,4 \times 10^{-30} \mathrm{C} \cdot \mathrm{m}$, que difere do valor conhecido $\left(\approx 6,1 \times 10^{-30} \mathrm{C} \cdot \mathrm{m}\right) \mathrm{em}$ aproximadamente $70 \% 17$

Antes de encerrarmos esta seção, vamos rediscutir a relação (33), mas agora substituindo (39) em (30) (para trabalharmos com $n$, em vez de $\alpha$ ), e também usando, em $(30)$, a relação $\pi a^{3}=3 /(4 N)$ (veja a nota de rodapé 13). Com essas substituições, obtemos

$$
\xi=\frac{2 \epsilon_{r}+\epsilon_{r} n^{2}}{2 \epsilon_{r}+n^{2}}
$$

Esta igualdade nos diz que $\xi$ não é muito maior que 1, mesmo se $\epsilon_{r} \gg n^{2}$ (como é o caso para água pura a $20^{\circ} \mathrm{C}$ ), pois neste caso temos $\xi \approx 1+n^{2} / 2$, e $n$ assume valores menores que 2 para a grande maioria dos líquidos. Por exemplo, para água pura a $20^{\circ} \mathrm{C}$ temos $n \approx 1,33 \mathrm{e}$, portanto, $\xi \approx 1,9$. E por que isso é importante? Porque como $\xi$ não é um fator muito maior que 1 , a condição de campos elétricos de baixa intensidade expressa em (33) corresponde à relação mais simples $p_{0} E \ll k T$.

\section{Modelo de Debye como resultado de uma aproximação de campo médio aplicada ao modelo de Onsager, e modelo de Clausius-Mossotti como caso particular dos modelos de Debye e Onsager}

A equação de Debye para fluidos polares puros [7] (aqui expressa no SI),

$$
\frac{\epsilon_{r}-1}{\epsilon_{r}+2}=\frac{N}{3 \epsilon_{0}}\left(\alpha+\frac{p_{0}^{2}}{3 k T}\right),
$$

\footnotetext{
17 Não se impressione com termos chegado à mesma ordem de grandeza, porque a equação de Debye 7]. (expressa em termos de $n$, em vez de $\alpha$ ), obtida 24 anos antes da equação de Onsager, nos fornece, com os mesmos valores para $\epsilon_{r}, n, N$ e $T, p_{0} \approx 2,7 \times 10^{-30} \mathrm{C} \cdot \mathrm{m}$.
} 
foi publicada 24 anos antes da equação de Onsager, e é citada por Onsager [1] logo no início de seu trabalho. De fato, o artigo de Onsager consiste essencialmente em uma crítica ao modelo de Debye, com a apresentação de um novo modelo.

A equação de Debye pode ser obtida através da mesma sequência de passos que apresentamos na seção 2. Basicamente, a única diferença está na expressão que Debye usou para o campo interno:

$$
\boldsymbol{E}_{\text {int }}^{\text {Debye }}=\left(\frac{\epsilon_{r}+2}{3}\right) \boldsymbol{E},
$$

em vez de $\boldsymbol{E}_{\text {int }}=\boldsymbol{R}+\boldsymbol{G}$ (veja a igualdade $(17)$ ). Se você conseguiu acompanhar os cálculos, apresentados neste artigo, que levaram à equação de Onsager (igualdade (40), certamente não terá dificuldade de obter a equação de Debye (igualdade (41)), porque a expressão para o campo interno de Debye deixa as contas muito mais simples. O que nos interessa, nesta seção, é (1) mostrar que o campo interno de Debye é a média do campo interno de Onsager (daí o título da seção), (2) mostrar qual é a origem da expressão usada por Debye para o campo interno (igualdade(42) - já que o trabalho de Debye é anterior ao de Onsager -, (3) mostrar por que se trata do mesmo campo, e (4) comparar as equações de Onsager e Debye.

(1) Temos, a partir de (17), 15 e 20 (nesta ordem):

$$
\begin{aligned}
\overline{\boldsymbol{E}_{\mathrm{int}}} & =\overline{\boldsymbol{G}}+\overline{\boldsymbol{R}} \\
& =\boldsymbol{G}+\overline{\boldsymbol{R}} \\
& =\left(\frac{3 \epsilon_{r}}{2 \epsilon_{r}+1}\right) \boldsymbol{E}+\frac{2}{3} \frac{\left(\epsilon_{r}-1\right)^{2}}{2 \epsilon_{r}+1} \boldsymbol{E} \\
& =\left(\frac{\epsilon_{r}+2}{3}\right) \boldsymbol{E} \\
& =\boldsymbol{E}_{\text {int }}^{\text {Debye }} .
\end{aligned}
$$

Ou seja, o campo interno de Debye é a média do campo interno de Onsager, e então podemos pensar o modelo de Debye como resultado de uma aproximação de campo médio aplicada ao modelo de Onsager.

(2) Agora, vejamos a origem da expressão em (42), sem referência ao campo interno de Onsager.

Voltemos ao fluido polar puro uniformemente polarizado ilustrado na Fig.2, Imagine ser possível "congelar a polarização do fluido", e então retirar do mesmo uma porção macroscópica no formato de uma esfera sem que a polarização além dessa esfera seja alterada (trata-se de uma experiência de pensamento). Com isso, fora da cavidade esférica macroscópica criada temos um fluido uniformemente polarizado, e isso significa que a densidade volumétrica de cargas ligadas, $\rho_{b}=-\boldsymbol{\nabla} \cdot \boldsymbol{P}$, é nula, restando então apenas densidades superficiais de cargas ligadas, $\sigma_{b}=\boldsymbol{P} \cdot \hat{\boldsymbol{n}}$ (lembrando que o versor $\hat{\boldsymbol{n}}$ é normal à superfície e aponta para fora do dielétrico), nas regiões do dielétrico em contato com as placas do capacitor e na superfície esférica (veja a Fig. 6). Obtemos o campo nessa cavidade macroscópica (nas condições expressas na experiência de pensamento acima - ou seja, mantida

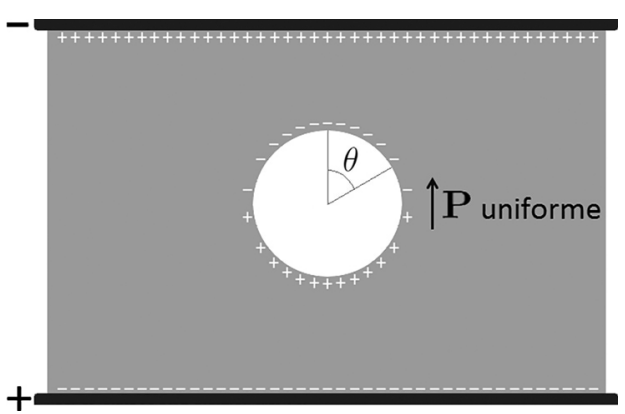

Figura 6: Cavidade esférica macroscópica criada na região central do dielétrico da Fig.2 A porção do fluido além da cavidade teve sua polarização elétrica previamente "congelada".

uniforme a polarização do fluido além da cavidade) como a soma do campo $\boldsymbol{E}_{\text {ext }}$ gerado pelas placas do capacitor, com o campo de módulo $\sigma_{b} / \epsilon_{0}=P / \epsilon_{0}$ (e que tem sentido oposto ao de $\boldsymbol{E}_{\text {ext }}$ ) gerado pelo acúmulo de cargas ligadas nas regiões do dielétrico em contato com as placas do capacitor, com o campo $\boldsymbol{E}_{\text {es } f}$ gerado pelo acúmulo de cargas ligadas na superfície esférica (Fig.66). Perceba que, devido ao congelamento da polarização, a soma dos dois primeiros campos é igual ao campo macroscópico uniforme $\boldsymbol{E}$ que havia antes do processo de congelamento da polarização do fluido e retirada de uma porção esférica macroscópica do mesmo. Temos então que o campo na cavidade macroscópica é igual a $\boldsymbol{E}+\boldsymbol{E}_{\text {esf }}$, e este foi o campo usado por Debye como campo interno, em seu modelo. Ou seja,

$$
\boldsymbol{E}_{\text {int }}^{\text {Debye }}=\boldsymbol{E}+\boldsymbol{E}_{e s f} .
$$

Obviamente, temos aqui o mesmo problema já apresentado neste artigo, ao transformarmos uma cavidade macroscópica em uma cavidade microscópica.

Calcular o campo $\boldsymbol{E}_{\text {esf }}$ no centro da cavidade esférica é tarefa simples. Temos, em coordenadas esféricas (com o ângulo $\theta$ indicado na Fig. 6),

$$
\sigma_{b}=\boldsymbol{P} \cdot \hat{\boldsymbol{n}}=-P \cos \theta,
$$

e então o elemento de carga

$$
\mathrm{d} q=\sigma_{b} \mathrm{~d} a=\sigma_{b} r^{2} \operatorname{sen} \theta \mathrm{d} \theta \mathrm{d} \phi=-P r^{2} \cos \theta \operatorname{sen} \theta \mathrm{d} \theta \mathrm{d} \phi,
$$

em que $r$ é o raio da esfera. A partir daqui, é realmente simples obtermos

$$
\boldsymbol{E}_{e s f}=\frac{\boldsymbol{P}}{3 \epsilon_{0}}
$$

Mais complicado é mostrar que esta expressão não se aplica apenas ao centro da cavidade esférica, mas que se trata de um campo elétrico uniforme. Substituindo 45 em (44), obtemos, então:

$$
\boldsymbol{E}_{\text {int }}^{\text {Debye }}=\boldsymbol{E}+\frac{\boldsymbol{P}}{3 \epsilon_{0}}=\boldsymbol{E}+\frac{\epsilon_{0} \chi_{e} \boldsymbol{E}}{3 \epsilon_{0}}=\left(\frac{\epsilon_{r}+2}{3}\right) \boldsymbol{E} .
$$

(3) Chegamos à mesma expressão, $\left(\epsilon_{r}+2\right) \boldsymbol{E} / 3$, em 43) e em (46), por dois caminhos bastante distintos. 
É importante enxergarmos por que se trata do mesmo campo.

Voltemos à experiência de pensamento que acabamos de descrever, mas, desta vez, imagine que congelamos a polarização apenas do volume esférico que foi retirado, deixando o restante do dielétrico livre. Com a retirada dessa esfera uniformemente polarizada, deixando em seu lugar uma cavidade esférica, as linhas de campo no dielétrico além da cavidade tendem a contorná-la, como ilustra a Fig.7 Reintroduzindo na cavidade a esfera uniformemente polarizada, recuperamos a polarização uniforme $\boldsymbol{P}$ inicial do dielétrico como um todo. Mas, nesse processo, o que importa não é a esfera uniformemente polarizada, em si, mas o campo que ela produz no resto do dielétrico, percebe? Isso significa que qualquer coisa que introduzamos na cavidade que produza o mesmo campo elétrico gerado por aquela esfera uniformemente polarizada nos fará recuperar a polarização uniforme inicial do meio além do volume esférico, que é a polarização ilustrada na Fig. 6. que, por sua vez, juntamente com o campo externo $\boldsymbol{E}_{\text {ext }}$ gera o campo interno de Debye. Agora, você sabia que o campo elétrico gerado por uma esfera de raio $r$ com polarização uniforme $\boldsymbol{P}$ é igual ao campo de um dipolo puro com momento de dipolo igual a $\left(4 \pi r^{3} / 3\right) \boldsymbol{P}$ ? (Veja, por exemplo, a seção 4.2.1 do Griffiths [2].) Então podemos recuperar a polarização uniforme $\boldsymbol{P}$ inicial além do volume esférico introduzindo no centro da cavidade um dipolo puro com momento de dipolo $\left(4 \pi r^{3} / 3\right) \boldsymbol{P}$, e o campo que age sobre esse dipolo é então campo interno de Debye. Ou seja, $\boldsymbol{E}_{\text {int }}^{\text {Debe }}$ é igual ao campo que age sobre um dipolo, com momento de dipolo $\left(4 \pi r^{3} / 3\right) \boldsymbol{P}$, no centro da cavidade esférica. Ora, mas este é o campo interno de Onsager (igualdade 14) com $\boldsymbol{p}=\left(4 \pi r^{3} / 3\right) \boldsymbol{P}$,

$$
\boldsymbol{E}_{\mathrm{int}}=\left(\frac{3 \epsilon_{r}}{2 \epsilon_{r}+1}\right) \boldsymbol{E}+\left(\frac{\epsilon_{r}-1}{2 \epsilon_{r}+1}\right) \frac{\left(4 \pi r^{3} / 3\right) \boldsymbol{P}}{2 \pi \epsilon_{0} a^{3}}
$$

que, por sua vez, é igual a $\overline{\boldsymbol{E}_{\text {int }}}$, pois

$$
\begin{aligned}
\overline{\boldsymbol{E}_{\mathrm{int}}} & =\overline{\boldsymbol{G}}+\overline{\boldsymbol{R}} \\
& =\left(\frac{3 \epsilon_{r}}{2 \epsilon_{r}+1}\right) \boldsymbol{E}+\left(\frac{\epsilon_{r}-1}{2 \epsilon_{r}+1}\right) \frac{\overline{\boldsymbol{p}}}{2 \pi \epsilon_{0} a^{3}}, \\
& =\left(\frac{3 \epsilon_{r}}{2 \epsilon_{r}+1}\right) \boldsymbol{E}+\left(\frac{\epsilon_{r}-1}{2 \epsilon_{r}+1}\right) \frac{\left(4 \pi r^{3} / 3\right) \boldsymbol{P}}{2 \pi \epsilon_{0} a^{3}}
\end{aligned}
$$

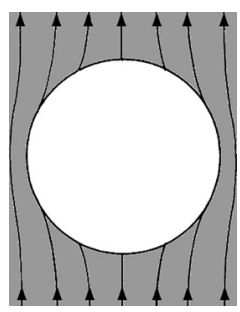

Figura 7: Linhas de campo em um dielétrico com uma cavidade esférica macroscópica e submetido a um campo elétrico externo uniforme. (veja as igualdades $(18)$ e $(19)$ ). Portanto, podemos enxergar fisicamente (matematicamente já mostramos) que $\boldsymbol{E}_{\text {int }}^{\text {Debye }}=\overline{\boldsymbol{E}_{\text {int }}^{-}}$.

(4) Agora, tratemos da comparação entre as equações de Onsager e Debye. Para isso, convém que essas equações sejam expressas com as mesmas quantidades: $\epsilon_{r}, N, T, \alpha$ e $p_{0}$, ou então $\epsilon_{r}, N, T, n$ e $p_{0}$ (ou seja, devemos escolher entre $\alpha$ e $n$ ). Já obtivemos uma versão para a equação de Onsager com as variáveis do primeiro grupo: a igualdade (38). Podemos reescrever a equação de Debye em termos das variáveis do segundo grupo substituindo (39) em (41). Após algumas manipulações algébricas simples, obtemos:

$$
\frac{3\left(\epsilon_{r}-n^{2}\right)}{\left(\epsilon_{r}+2\right)\left(n^{2}+2\right)}=\frac{N}{3 \epsilon_{0}} \frac{p_{0}^{2}}{3 k T} .
$$

Podemos então comparar as equações (38) e (41), ou as equações (40) e 47). Perceba que estas duas últimas igualdades são particularmente adequadas quando queremos prever (segundo o modelo considerado, claro) o valor de $p_{0}$ a partir de medições macroscópicas: de $\epsilon_{r}$, $N, T$ e $n$. Já as duas primeiras são próprias se queremos prever $\epsilon_{r}$ a partir do conhecimento dos valores de $N, T$, $\alpha$ e $p_{0}$ para o fluido sob investigação. No primeiro caso "caminhamos do macro para o micro", e, no segundo, "caminhamos do micro para o macro".

Há muitas formas de compararmos as equações de Onsager e Debye, mas, seja qual for a escolha, devemos lembrar que a diferença fundamental entre os modelos de Onsager e Debye está na expressão usada para o campo interno, e que o campo interno de Debye é a média do campo interno de Onsager. Assim, as diferenças ou semelhanças entre as equações de Onsager e Debye devem ser interpretadas a partir dessa relação entre seus campos internos.

Por exemplo, no caso particular em que $p_{0}=0$ (moléculas apolares), o campo de reação $\boldsymbol{R}$ não muda com as rotações da molécula considerada (veja a igualdade (21) ), e, assim, o campo interno de Onsager é igual à sua própria média, e, portanto, é igual ao campo interno de Debye (veja a igualdade 43 ). Então não pode haver diferença entre as equações de Onsager e Debye, nesse caso particular; e, de fato, não há, como podemos concluir fazendo $p_{0}=0 \mathrm{em}$ e 441 . O caso particular das equações de Onsager e Debye em que $p_{0}=0$,

$$
\frac{\epsilon_{r}-1}{\epsilon_{r}+2}=\frac{N \alpha}{3 \epsilon_{0}}
$$

é conhecido como "equação de Clausius-Mossotti".

Como um segundo exemplo, podemos resolver as equações (38), (41) e (48) para $\epsilon_{r}$, e então traçar em uma mesma figura os três gráficos de $\epsilon_{r}$ versus $N$, partindo de $N=0$, com uma escolha apropriada de valores para os demais parâmetros. Na Fig. 8 trabalhamos com a polarizabilidade e o momento de dipolo permanente do $\mathrm{HBr}$, e com $T=200 \mathrm{~K}$. Nosso interesse é tão somente comparar o que preveem os três modelos. Observe que os modelos de Onsager e Debye fornecem para $\epsilon_{r}$ valores muito próximos entre si quando $N$ é próximo de 0 , e, é 


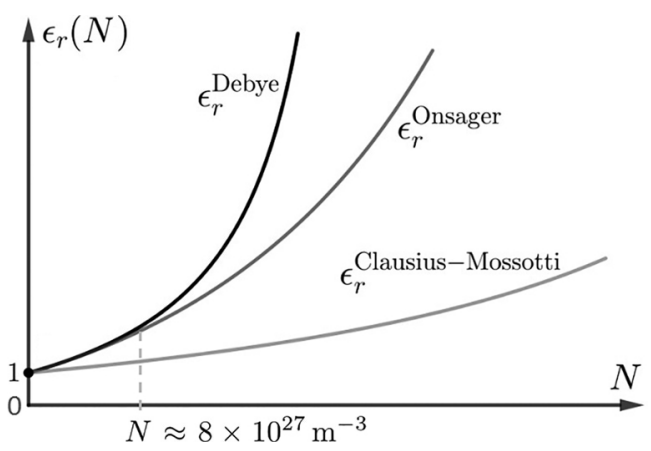

Figura 8: Gráficos de $\epsilon_{r} \times N$ a partir das equações de Onsager, Debye e Clausius-Mossotti, considerando-se a polarizabilidade e o momento de dipolo permanente do $\mathrm{HBr}$, e $T=200 \mathrm{~K}$.

claro, nos três casos $\epsilon_{r}$ tende a 1 quando $N$ tende a 0 (afinal, a constante dielétrica do vácuo é 1). Em termos da análise dos campos internos, essa proximidade entre os valores previstos para $\epsilon_{r}$ pelos modelos de Onsager e Debye, no caso de baixas densidades, é esperada, porque a relação $N \approx 1 /\left(4 \pi a^{3} / 3\right)$ (veja a nota de rodapé 13$)$ nos dá, para valores pequenos de $N$, valores elevados para $a^{3}$, e, portanto, baixas intensidades do campo de reação (veja a igualdade $(16)$ ). Como $\overline{\boldsymbol{G}}=\boldsymbol{G}$, a diferença entre o campo interno de Onsager e seu valor médio, valor médio este que é igual ao campo interno de Debye, existe devido à diferença entre $\boldsymbol{R}$ e $\overline{\boldsymbol{R}}$. Quando a intensidade de $\boldsymbol{R}$ tende a 0 , a diferença entre $\boldsymbol{R}$ e $\overline{\boldsymbol{R}}$ também tende a 0 , e, portanto, tende a 0 a diferença entre os campos internos de Onsager e Debye. O resultado esperado é então que os dois modelos nos deem para $\epsilon_{r}$ valores próximos, no caso de baixas densidades. Vamos propor a você que explique por que temos $\epsilon_{r}^{\text {Debye }}>\epsilon_{r}^{\text {Onsager }}>\epsilon_{r}^{\text {Clausius-Mossotti, }}$ e por que a curva $\epsilon_{r}^{\text {Clausius-Mossotti }} \times N$ não se aproxima muito das outras duas na faixa de valores de $N$ em que $\epsilon_{r}^{\text {Debye }}$ e $\epsilon_{r}^{\text {Onsager }}$ se confundem.

\section{Conclusão}

Neste artigo, guiamos o leitor ou a leitora à obtenção da equação de Onsager (Equation (40)), e no percurso fizemos discussões, relativas a modelagem em física, que são de interesse geral na formação de profissionais da área. Esperamos ter ajudado a mostrar um pouco de como os físicos trabalham, e sugerimos o uso deste texto no estudo ou ensino de tópicos complementares em disciplinas de eletrodinâmica clássica, mecânica estatística ou físicoquímica, em cursos de graduação ou pós-graduação.

Mas, é claro, o modelo de Onsager para fluidos polares puros tem importância em si mesmo, e acreditamos que este texto será útil também àqueles que realizarão atividades de pesquisa que, de alguma forma, envolvem esse modelo - ou mesmo parte dele, como o conceito de campo de reação (como é o caso, por exemplo, quando se estuda o chamado efeito do solvente sobre a estrutura eletrônica de moléculas). Apreender o modelo de Onsager através deste artigo é, acreditamos, tarefa bem mais simples que através da leitura do artigo original [1]. Não obstante, saiba que o artigo original realiza certas discussões e desenvolve certos tópicos que não abordamos aqui, como, por exemplo, constante dielétrica de soluções. Ao mesmo tempo, realizamos discussões que não estão presentes no artigo de Onsager.

Até a data da submissão deste trabalho, o artigo de Onsager [1] tinha sido citado por outros 4325 artigos - o primeiro ainda em 1936. Foram 23 citações em 2019, e já constavam 11 citações em 2020 18

Discutimos também o modelo de Debye, mas de uma forma que consideramos bastante interessante: como resultado de uma aproximação de campo médio aplicada ao modelo de Onsager. Essa abordagem deixa mais clara e simples a comparação entre os dois modelos. E, entre outras coisas, mostramos que ambos os modelos recaem no modelo de Clausius-Mossotti no caso particular de moléculas apolares.

Uma sequência natural a partir deste artigo, para os que se interessam por teoria de polarização elétrica, consiste no estudo do modelo de Kirkwood-Fröhlich, que considera interações de curto alcance em fluidos polares puros, e, como dissemos, foi desenvolvido (inicialmente por Kirkwood [4], tendo recebido depois a contribuição de Fröhlich [5]) a partir do modelo de Onsager.

\section{Agradecimentos}

Agradecemos à FACEPE pela bolsa de Iniciação Científica concedida a Adonias Barros, e aos professores Mário Henrique B. G. Oliveira, da UFRPE, e Gustavo Camelo Neto, da UFPE, pela leitura crítica do artigo.

\section{Material Suplementar}

O seguinte material suplementar está disponível online: Aproximação para a função de Langevin (relação (34) do artigo principal).

\section{Referências}

[1] L. Onsager, Journal of the American Chemical Society 58, 1486 (1936).

[2] D.J. Griffiths, Introduction to Electrodynamics (Cambridge University Press, Cambridge, 2017), $4^{a}$ ed.

[3] F. Reif, Fundamentals of Statistical and Thermal Physics (Waveland Press, Long Grove, 2008).

[4] J.G. Kirkwood, The Journal of Chemical Physics 7, 911 (1939).

[5] H. Fröhlich, Theory of Dielectrics - Dielectric Constant and Dielectric Loss (Clarendon Press, Oxford, 1949).

[6] J.D. Jackson, Classical Electrodynamics (John Wiley, New York, 1999), $3^{a}$ ed.

[7] P. Debye, Physik. Zeits. 13, 97 (1912).

${ }_{18}$ Para obter números atualizados, acesse: https://pubs.acs.org/doi/abs/10.1021/ja01299a050 